\title{
Distribution of pomalidomide into semen of healthy male subjects after multiple doses
}

This article was published in the following Dove Press journal:

Clinical Pharmacology:Advances and Applications

\author{
Yan $\mathrm{Li}^{1}$ \\ Xiaomin Wang ${ }^{2}$ \\ Liangang Liu $^{3}$ \\ Josephine Reyes' \\ Maria Palmisano' \\ Simon Zhou'
}

'Translational Development and Clinical Pharmacology, Celgene

Corporation, Summit, NJ, USA;

${ }^{2}$ Non-Clinical Development, Celgene

Corporation, Summit, NJ, USA;

${ }^{3}$ Biometrics and Data Operations,

Celgene Corporation, Summit, NJ,

USA
Correspondence: Simon Zhou

Translational Development and Clinical

Pharmacology, Celgene Corporation, 86

Morris Avenue, Summit, NJ 07920, USA

Tel + I 9086739284

Fax + I 9086732842

Email szhou@celgene.com
Objective: To assess whether pomalidomide can distribute into human semen and its duration in human semen.

Method: A phase 1, randomized, double-blind, placebo-controlled study (CC-4047-CP-006) was conducted to evaluate the safety, tolerability, and pharmacokinetics of pomalidomide (CC4047) following multiple daily doses in healthy male subjects. Semen samples were collected on Day -1 and 4 hours after dosing on Day 4 to quantify the pomalidomide concentrations in ejaculate after multiple oral doses of pomalidomide.

Result: Our study showed that pomalidomide was present in male subjects' semen samples, and the average amount of pomalidomide in a single ejaculate 4 hours after dosing was less than $0.0022 \%$ of the daily $2 \mathrm{mg}$ dose. There was a good correlation between the semen concentrations and the plasma concentrations, suggesting that the plasma concentration may be the main driving force for the distribution of pomalidomide into the seminal reservoirs. Simulation results suggest that pomalidomide was undetectable in semen 48 hours after stopping dosing. Conclusion: Based on the results from this study, the pomalidomide prescribing information approved by the US Food and Drug Administration includes a statement that "pomalidomide is present in the semen of patients receiving the drug. Therefore, males must always use a latex or synthetic condom during any sexual contact with females of reproductive potential while taking POMALYST and for up to 4 weeks after discontinuing POMALYST, even if they have undergone a successful vasectomy. Male patients taking POMALYST must not donate sperm." Keywords: pomalidomide, distribution, semen, teratogen, kinetics

\section{Introduction}

Pomalidomide is an immunomodulatory agent with antineoplastic activity. ${ }^{1,2}$ In in vitro cellular assays, pomalidomide inhibited proliferation and induced apoptosis of hematopoietic tumor cells ${ }^{3-7}$ and showed immunomodulatory activity. ${ }^{8-10}$ The dosage of $4 \mathrm{mg}$ per day taken orally on Days 1-21 of repeated 28-day cycles was approved (in combination with dexamethasone) in the European Union and United States for the treatment of patients with multiple myeloma who have received $\geq 2$ prior therapies, including lenalidomide and bortezomib (in the European Union; a proteasome inhibitor in the United States), and who have progressed on or within 60 days of completion of the last therapy or had disease progression while on their last therapy. ${ }^{11,12}$

IMiD immunomodulatory agents, including lenalidomide and pomalidomide, are a class of drugs structurally similar to thalidomide, which has long been known as a human teratogen and causes severe developmental deformities, including 
phocomelia. ${ }^{13-15}$ In the preclinical $\left[{ }^{14} \mathrm{C}\right]$ thalidomide study, radioactivity was found in rabbit semen, and congenital malformations were observed in newborn offspring of male rabbits that had received thalidomide for prolonged periods of time. ${ }^{16-18}$ Although such a congenital malformation effect has not been observed or confirmed in humans, thalidomide has been shown to distribute into human semen, ${ }^{19}$ and the potential risk of embryo/fetal exposure to thalidomide through semen transmission could not be ruled out. In addition, Chen et a ${ }^{16}$ showed in an embryo/fetal development study in rhesus monkeys that lenalidomide produced malformations in the offspring of female monkeys that received the drug during pregnancy. Chen et a ${ }^{16}$ also showed that lenalidomide is present in human semen. Thus, the teratogenic effect of lenalidomide in humans cannot be ruled out.

The structural similarity of pomalidomide to thalidomide and lenalidomide suggests a similar potential risk to the developing embryo or fetus. In a fertility and early embryonic development study in rats, pomalidomide doses of $25 \mathrm{mg} / \mathrm{kg} / \mathrm{d}$ or higher in males and females resulted in a decrease in mean number of viable embryos and an increase in postimplantation loss. ${ }^{11,12}$ In addition, pomalidomide was found to be teratogenic in both rats and rabbits when administered during the period of major organogenesis. ${ }^{11,12}$ Pomalidomide showed good distribution properties into most tissues and organs in preclinical absorption, distribution, metabolism, and excretion studies: following a single oral administration of $\left[{ }^{14} \mathrm{C}\right]$-pomalidomide to pigmented male Long-Evans rats, pomalidomide-associated radioactivity was rapidly (time to maximum plasma concentration $\left[t_{\text {max }}\right]$ of 3 hours in most tissues) and widely distributed in tissues, and moderate concentrations were found in most tissues, including spinal cord and brain (data on file). Therefore, whether pomalidomide can distribute into human semen and its duration in semen are clinically relevant issues pertinent to the potential transmission of pomalidomide through semen to male subjects' female partners of childbearing potential.

CC-4047-CP-006 was a phase 1, randomized, doubleblind, placebo-controlled study to evaluate the safety, tolerability, and pharmacokinetics (PK) of pomalidomide (CC-4047) following multiple daily doses in healthy male subjects. The primary objectives of the study were to evaluate the safety and tolerability of pomalidomide administered orally once a day (QD) for 5 days, at daily doses of $0.5 \mathrm{mg}$, $1 \mathrm{mg}$, or $2 \mathrm{mg}$, and to determine the PK of pomalidomide after multiple daily doses for 5 days. The secondary objective was to quantify the pomalidomide concentrations in semen ejaculates after multiple oral doses of pomalidomide in healthy male subjects. The study consisted of 3 sequentially enrolled cohorts. Satisfactory safety data from the previous dose level allowed the initiation of the next cohort with a higher daily dose of pomalidomide. Serial blood samples for PK and pharmacodynamic (PD) analysis were collected during the study, and semen samples were collected from subjects in the $2 \mathrm{mg}$ dose cohort. Safety was monitored throughout the study. Results from this study allowed a preliminary assessment and a better understanding of the kinetics and distribution of pomalidomide into human semen.

\section{Methods}

All subjects provided written informed consent prior to screening. This study was conducted and monitored in accordance with Celgene procedures and the study protocol, which complied with the ethical principles of the International Conference on Harmonisation Guideline for Good Clinical Practice E6 (R1), as required by the major regulatory authorities. The conduct also complied with the Declaration of Helsinki, Title 21 of the US Code of Federal Regulations, Parts 50 and 56 , concerning informed consent, as well as independent review board regulations and applicable national, state, and local laws or regulations. This study was conducted by PPD Development (Austin, TX, USA) and was approved by the Salus independent review board (Austin, TX, USA).

\section{Study design}

This was a single-center, randomized (within each dose level), placebo-controlled, double-blind, multiple ascending dose study in healthy male subjects. A total of 33 healthy men between 18 and 55 years of age, inclusive, were enrolled.

The study consisted of 3 sequentially enrolled cohorts. Satisfactory safety data from the previous cohort allowed the initiation of the next cohort using a higher daily dose of pomalidomide. Depending on the cohort they were enrolled in, participants received 1 of 3 doses of pomalidomide, or its matched placebo, in a single capsule QD for 5 days under fasted conditions: Cohort A, $0.5 \mathrm{mg}$ pomalidomide or placebo; Cohort B (after satisfactory safety review for Cohort A), $1 \mathrm{mg}$ pomalidomide or placebo; or Cohort C (after satisfactory safety review for Cohort B), $2 \mathrm{mg}$ pomalidomide or placebo.

Before the start of each cohort, there was a screening phase of up to 21 days during which subjects were evaluated for eligibility. Qualified subjects were admitted into the clinic on the day before dosing (Day -1) for baseline assessments and to confirm eligibility. In the morning of Day 1, following an overnight fast of at least 8 hours, eligible subjects received their randomization numbers and study drug. Subjects were confined at the clinic from Day 
-1 through Day 8 for study treatment, safety assessments, and serial PK and PD sampling. Subjects were discharged from the clinic on the morning of Day 8 upon completion of study procedures. A follow-up visit for safety evaluation occurred approximately 5-10 days after discharge on Day 8 or the day of early discontinuation.

Safety was monitored throughout the study. Safety evaluations included adverse event (AE) monitoring, clinical laboratory tests, vital sign measurements, 12-lead electrocardiograms (ECGs), and physical examinations. Blood samples were collected at predetermined times for PK and $\mathrm{PD}$ analysis. Semen samples were collected at predetermined times for PK analysis in Cohort $\mathrm{C}$ only.

\section{Blood and semen sample collection}

To determine pomalidomide concentrations in plasma, blood samples $(5 \mathrm{~mL})$ were collected on Day 1 before dosing (0 hour) and at $0.5,1,1.5,2,2.5,3,4,6,8,12$, and 24 hours after dosing; on Day 5 before dosing ( 0 hour) and at $0.5,1$, $1.5,2,2.5,3,4,6,8,12,24,36,48$, and 72 hours after dosing; and on Days 3 and 4 before dosing ( 0 hour).

To determine pomalidomide concentrations in semen, samples were collected from subjects on Day -1 and 4 hours after dosing on Day 4. Semen collection time was defined as the ending time of ejaculation. To assess potential contamination by urine in the Day 4 semen sample, the time at which subjects urinated between dosing and semen sample collection was to be recorded in source documents and the case report form.

\section{Bioanalytical methods}

Validated liquid chromatography-tandem mass spectrometry (LC-MS/MS) assays were used to assess pomalidomide concentrations in the plasma and semen samples. ${ }^{20}$ As an internal standard, plasma and semen samples were spiked with stable ${ }^{13} \mathrm{C}_{5}$-labeled pomalidomide. Pomalidomide and ${ }^{13} \mathrm{C}_{5}$-labeled pomalidomide were extracted using liquid-liquid extraction from $0.1 \mathrm{~mL}$ of acidified human semen samples (acidified with 1 volume of Sorenson's buffer) and $0.2 \mathrm{~mL}$ of acidified human plasma samples (acidified with citric acid). After transfer to a new tube, the solvent was evaporated, and the samples were reconstituted and injected for LC-MS/MS analysis using a Chiral AGP $(50 \times 9 \times 3.0 \mathrm{~mm}, 5$ Im; Chrom Tech, Inc., Apple Valley, MN, USA) analytical column. Positive ions were measured in the multiple reaction monitoring mode $(\mathrm{m} / \mathrm{z}$ of $274.1 / 201.1$ for pomalidomide and 279.1/204.0 for the internal standard) using a SciexAPI-4000 tandem mass spectrometer (Applied Biosystems, Foster City, CA, USA) equipped with a Turbo Ion Spray source. For the quality control samples, the accuracy ranged from $91.0 \%$ to $106.9 \%$. The pomalidomide standard curves were linear ( $r^{2}>0.99$ ), from 0.25 to $100 \mathrm{ng} / \mathrm{mL}$ for plasma and 0.5 to 100 $\mathrm{ng} / \mathrm{mL}$ for semen. The lower limit of quantitation was 0.25 $\mathrm{ng} / \mathrm{mL}$ for plasma and $0.5 \mathrm{ng} / \mathrm{mL}$ for semen. Plasma quality control samples at 3 concentrations $(0.75,40$, and $80 \mathrm{ng} / \mathrm{mL})$ were used to determine assay accuracy and precision. Semen quality control samples at 3 concentrations $(1.50,40$, and 80 $\mathrm{ng} / \mathrm{mL}$ ) were used to determine assay accuracy and precision. Interday assay accuracy ranged from -0.48 to 9.67 , deviating from the nominal concentration for semen sample analysis. Similarly, interday assay accuracy ranged from -5.21 to -3.47 , deviating from the nominal concentration for plasma sample analysis. Interday precision did not exceed $6.49 \%$ for either semen or plasma assays.

\section{PK analyses}

Pomalidomide plasma concentrations were measured at various time points to characterize the following PK parameters. For Day 1, area under the plasma drug concentration-time curve from time 0 to 24 hours $\left(\mathrm{AUC}_{0-24}\right)$, maximum observed plasma concentration $\left(C_{\max }\right)$, and time to $C_{\max }\left(t_{\max }\right)$ and for Day 5, AUC from time 0 to the last measurable concentration $\left(\mathrm{AUC}_{0-t}\right), \mathrm{AUC}_{0-24}, C_{\max }, t_{\max }$, terminal half-life $\left(t_{1 / 2}\right)$, apparent plasma clearance after oral administration (CL/F), apparent terminal volume of distribution after oral administration $(\mathrm{Vz} / \mathrm{F})$, and accumulation ratio. Plasma concentrations of pomalidomide were listed and summarized using descriptive statistics. Individual and mean concentration-versus-time profiles were generated. Pomalidomide plasma PK parameters were calculated using noncompartmental methods and actual blood sampling times with Phoenix ${ }^{\mathrm{TM}}$ WinNonlin $\AA$ Professional Version 6.3 (Pharsight ${ }^{\circledR}$, a Certara ${ }^{\mathrm{TM}}$ company, St. Louis, MO, USA).

The amount of pomalidomide that was excreted unchanged in the ejaculation was calculated as drug concentration in semen multiplied by the volume of ejaculate. The concentration values in semen samples below the lower limit of quantification (BLQ) were treated as 0 for the calculation of the total drug amount in an ejaculation. Percentage of dose excreted in each ejaculation from each subject was calculated as total amount of pomalidomide in ejaculate divided by the $2 \mathrm{mg}$ daily dose times 100 .

\section{Safety assessment}

Safety was monitored throughout the study. Safety evaluations include AE reporting, physical examinations, vital sign measurements, 12-lead ECGs, and clinical laboratory 
safety tests. All concomitant medications were assessed and recorded throughout the study from the time the informed consent document was signed until study completion (followup safety telephone call). AEs and serious AEs (SAEs) were assessed and recorded from the time the subject signed the informed consent document until study completion (followup safety telephone call), and when made known to the investigator within 28 days after the last dose of study drug (and those SAEs made known to the investigator at any time thereafter that were suspected to be related to the study drug).

\section{Results}

\section{Demographic and other baseline characteristics}

A total of 33 subjects were enrolled, and 31 (93.9\%) completed the study. Demographic data are summarized in Table 1. Overall, demographic characteristics were similar across treatments (Table 1). All subjects were men between 20 and 51 years old. Subjects' weight ranged from 63.1 to $94.7 \mathrm{~kg}$, height ranged from 161.9 to $184.0 \mathrm{~cm}$, and body mass index ranged from 20.6 to $29.9 \mathrm{~kg} / \mathrm{m}^{2}$. The majority of subjects were white (78.8\%) and not Hispanic or Latino (66.7\%).

\section{Pomalidomide PK in plasma}

Mean concentration-time profiles are presented in Figure 1. Following the $0.5 \mathrm{mg}$ oral dose administration, plasma concentration-time profiles on Day 1 (single dose) and Day 5 (multiple doses) were comparable in shape, with slightly higher mean concentrations on Day 5 versus Day 1. Similar PK profiles on Day 1 and 5 were noted at the 1.0 and $2.0 \mathrm{mg}$ dose levels. In all 3 dose cohorts, pomalidomide was readily absorbed and reached $C_{\max }$ between 1.50 and 2.25 hours after dosing. Then plasma concentrations declined in an apparent monophasic manner and were BLQ (ie, $0.250 \mathrm{ng} / \mathrm{mL}$ ) by 36 hours after the last dose on Day 5 of the 0.5 and $1.0 \mathrm{mg}$ doses and by 48 hours after the last $2.0 \mathrm{mg}$ dose.

PK plasma parameters for pomalidomide are presented in Table 2. Following the $0.5,1.0$, and $2.0 \mathrm{mg}$ pomalidomide doses, the geometric mean $\mathrm{AUC}_{0-24}$ on Day 5 were $66.5,103$, and $242 \mathrm{ng} \mathrm{h} / \mathrm{mL}$, respectively. At all 3 dose levels, the $\mathrm{AUC}_{0-24}$ was slightly higher (an increase of $4.0 \%-13.0 \%$ in geometric mean) on Day 5 than Day 1 (Table 2). The $C_{\max }$ values were also slightly higher (an increase of $4.0 \%-14.0 \%$ in geometric mean) on Day 5 than on Day 1 (Table 2). The $t_{1 / 2}, \mathrm{CL} / \mathrm{F}$, and $\mathrm{Vz} / \mathrm{F}$ were similar and consistent among the 3 dose levels.

\section{Pomalidomide quantities in semen}

Semen samples were collected from the 8 subjects enrolled in the $2 \mathrm{mg}$ cohort within or near the predefined time ( 4 hours after dosing on Day 4). The actual semen sample collection time ranged from 3.57 to 3.97 hours after doing, that is, 25.8-1.8 minutes before the predefined time. However, no

Table I Demographic summary

\begin{tabular}{|c|c|c|c|c|c|}
\hline \multirow[t]{2}{*}{ Demographics } & \multicolumn{4}{|l|}{ Treatment } & \multirow[t]{2}{*}{ Total $(\mathbf{N}=33)$} \\
\hline & $\begin{array}{l}\text { Pomalidomide } 0.5 \\
\mathrm{mg}(\mathrm{N}=8)\end{array}$ & $\begin{array}{l}\text { Pomalidomide I } \\
\mathrm{mg}(\mathrm{N}=\mathbf{8})\end{array}$ & $\begin{array}{l}\text { Pomalidomide } 2 \\
\mathrm{mg}(\mathrm{N}=8)\end{array}$ & $\begin{array}{l}\text { Placebo } \\
(\mathrm{N}=9)\end{array}$ & \\
\hline Age (years) & $32(22-44)$ & $34(26-45)$ & $30(20-42)$ & $33(2 \mid-5 I)$ & $32(20-5 I)$ \\
\hline \multicolumn{6}{|l|}{ Mean (range) } \\
\hline Gender, n (\%) & $8(100.0)$ & $8(100.0)$ & $8(100.0)$ & $9(100.0)$ & $33(100.0)$ \\
\hline \multicolumn{6}{|l|}{ Male } \\
\hline Race, n (\%) & $5(62.5)$ & 7 (87.5) & $6(75.0)$ & $8(88.9)$ & $26(78.8)$ \\
\hline \multicolumn{6}{|l|}{ White } \\
\hline Race, n (\%) & $3(37.5)$ & I (12.5) & I (I2.5) & I (II.I) & $6(18.2)$ \\
\hline \multicolumn{6}{|l|}{ Black or African American } \\
\hline Race, n (\%) & 0 & 0 & $\mathrm{I}(12.5)$ & 0 & I (3.0) \\
\hline \multicolumn{6}{|c|}{ Native Hawaiian or other Pacific } \\
\hline \multicolumn{6}{|l|}{ Islander } \\
\hline Ethnicity, n (\%) & $4(50.0)$ & I (12.5) & $4(50.0)$ & $2(22.2)$ & II (33.3) \\
\hline \multicolumn{6}{|l|}{ Hispanic or Latino } \\
\hline Ethnicity, n (\%) & $4(50.0)$ & $7(87.5)$ & $4(50.0)$ & 7 (77.8) & $22(66.7)$ \\
\hline \multicolumn{6}{|l|}{ Not Hispanic or Latino } \\
\hline Weight (kg) & 79.4 (63.6-94.7) & $78.4(68.1-88.1)$ & 76.1 (63.1-88.9) & 76.1 (63.5-92.4) & 77.4 (63.1-94.7) \\
\hline \multicolumn{6}{|l|}{ Mean (range) } \\
\hline Height $(\mathrm{cm})$ & I73.| (I63.9-|83.0) & $174.6(166.5-184.0)$ & $174.0(165.1-180.0)$ & $175.6(|6| .9-183.5)$ & I74.4 (I6I.9-184.0) \\
\hline \multicolumn{6}{|l|}{ Mean (range) } \\
\hline Body mass index $\left(\mathrm{kg} / \mathrm{m}^{2}\right)$ & $26.5(22.8-29.0)$ & $25.8(21.2-28.9)$ & $25.2(20.6-29.9)$ & $24.6(21.4-28.2)$ & $25.5(20.6-29.9)$ \\
\hline Mean (range) & & & & & \\
\hline
\end{tabular}



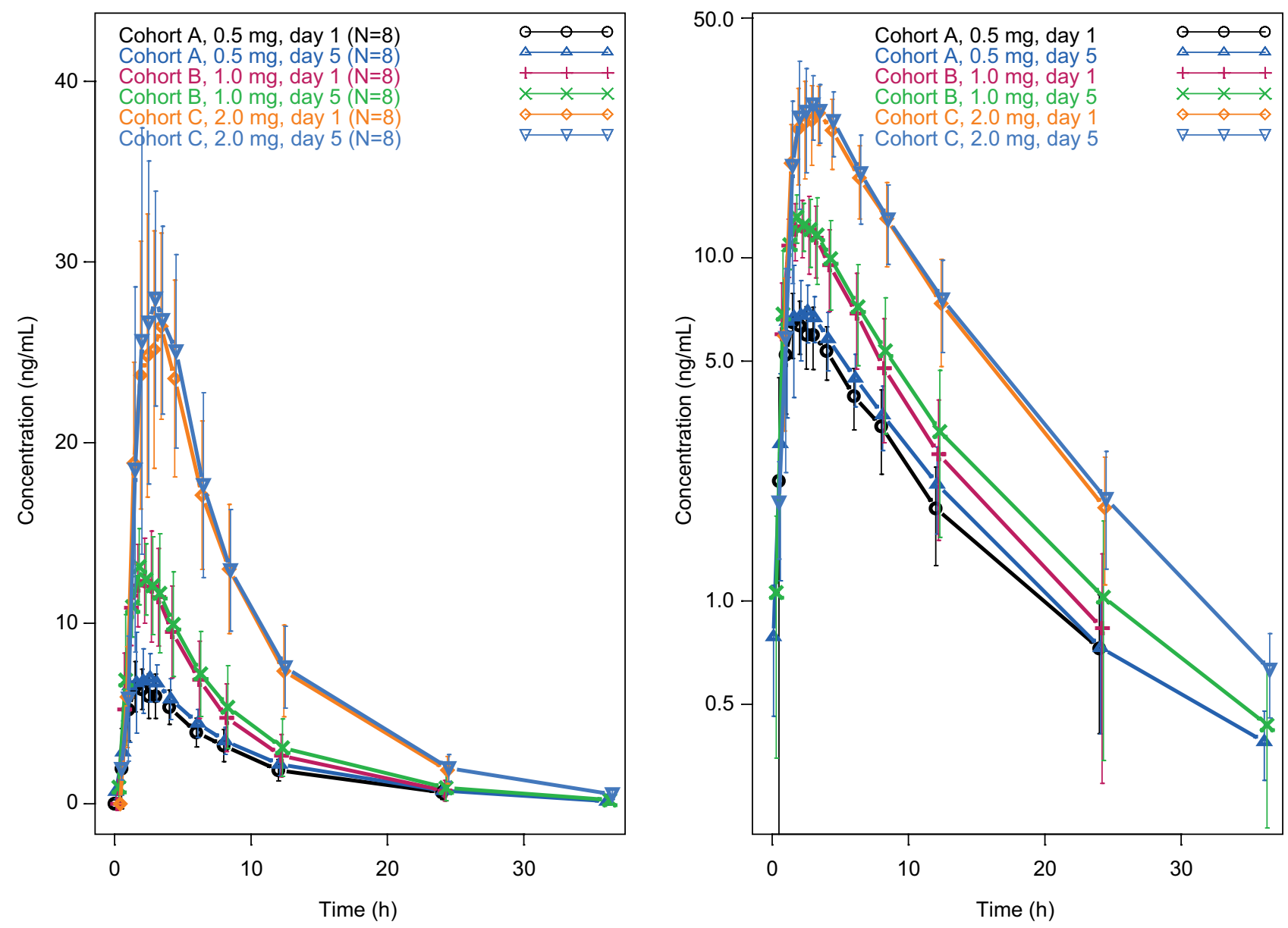

Figure I Mean $( \pm S D)$ pomalidomide plasma concentration profiles following single- and multiple-dose administration (left panel: linear scale; right panel: semi-log scale). Abbreviation: SD, standard deviation.

considerable differences were observed in the semen concentration values from these subjects' samples.

The concentration-versus-time plot indicates that pomalidomide was present in semen 4 hours after oral administration (Figure 2). The semen concentrations were more than half of the corresponding plasma concentrations; individual semen concentrations following the $2 \mathrm{mg}$ pomalidomide doses are presented in Table 3. The amount of pomalidomide recovered in semen samples 4 hours after dosing (on Day 4) ranged from 10.5 to $113 \mathrm{ng}$, with a geometric mean concentration of $16.4 \mathrm{ng} / \mathrm{mL}$, which was approximately $67.0 \%$ of pomalidomide plasma concentration observed at the same time point on Day 5 (ie, $24.5 \mathrm{ng} / \mathrm{mL}$ ) suggesting a good distribution of pomalidomide into semen. The maximum drug amount in a single ejaculate was $113 \mathrm{ng}(0.0057 \%$ of the dose), and the average amount of pomalidomide in ejaculate 4 hours after dosing was equivalent to $0.0022 \%$ of the daily $2 \mathrm{mg}$ dose, which was considered a minor portion of the daily dose.
In general, there was good correlation between the semen concentrations ( 4 hours after the Day 4 dose) and the plasma concentrations (4 hours after the Day 1 and Day 5 doses), that is, the subjects who had higher plasma pomalidomide concentrations 4 hours after the Day 1 or Day 5 doses tended to have higher semen pomalidomide concentrations 4 hours after the Day 4 dose (Figure 3) suggesting that the plasma concentration may be the main driving force for the distribution of pomalidomide into the seminal reservoirs.

In a previous study assessing the distribution of lenalidomide (an analog of pomalidomide with a similar chemical structure) into semen in healthy men, ${ }^{16}$ the disappearance rate constant of lenalidomide from the semen samples and plasma samples was quantitatively similar. In addition, in the distribution study in rats, it was shown that following a single oral administration of $\left[{ }^{14} \mathrm{C}\right]$-pomalidomide to pigmented male Long-Evans rats, the terminal elimination rate constants of pomalidomide from the reproductive tissues (epididymis, prostate gland, and seminal vesicles) were comparable to 
those from the plasma. Therefore, the simulation of the semen concentration profiles was conducted assuming that the elimination rate constant from the semen is the same as that from the plasma (Figure 4). Simulation results suggest that the semen concentrations would be BLQ $(0.5 \mathrm{ng} / \mathrm{mL})$ less than 48 hours after dosing.

\section{Safety}

Pomalidomide administered orally QD for 5 days at daily doses of $0.5,1$, or $2 \mathrm{mg}$ was safe and well tolerated by the study subjects. There were no deaths, SAEs, or AEs leading to discontinuation. All treatment-emergent AEs were mild in severity, were not suspected to be related to study drug, and resolved by the end of the study. There was no apparent treatment- or dose-related trend in clinical laboratory results, vital sign measurements, 12-lead ECG results, or physical examination findings. No subject had a clinical laboratory, vital sign, or safety ECG result considered clinically significant or reported as a treatment-emergent AE.

\section{Discussion}

Thalidomide caused a disaster that still shocks the world today. Exposure to the drug during early embryonic development resulted in a range of damage, including severe developmental deformities such as phocomelia. ${ }^{13-15}$ The mechanisms underlying thalidomide's teratogenic actions remain controversial. It has been suggested that the drug targets multiple tissues by affecting a global process, such as angiogenesis. D'Amato et $\mathrm{al}^{21}$ showed that thalidomide inhibits the angiogenic vascularization of rodent corneas induced by fibroblast growth factor protein. This discovery led to the theory that thalidomide might cause its teratogenic damage by targeting embryonic blood vessels. ${ }^{13}$

The similarity of pomalidomide's structure to that of its analog thalidomide suggests a potential risk to the developing embryo or fetus. In a fertility and early embryonic development study in rats, pomalidomide treatment of males and females resulted in a decrease in mean number of viable embryos and an increase in postimplantation loss. Pomalidomide was found to be teratogenic in both rats and rabbits when administered during the period of major organogenesis, ${ }^{11,12}$ and the teratogenic effect of pomalidomide in humans cannot be ruled out.

Human ejaculate is primarily composed of secretions from testes, seminal vesicles, and the prostate. Secretions from the urethral and bulbourethral glands, epididymis, and ampullae contribute an additional $10 \%$. Although many studies have been devoted to drug effects on spermatozoal 


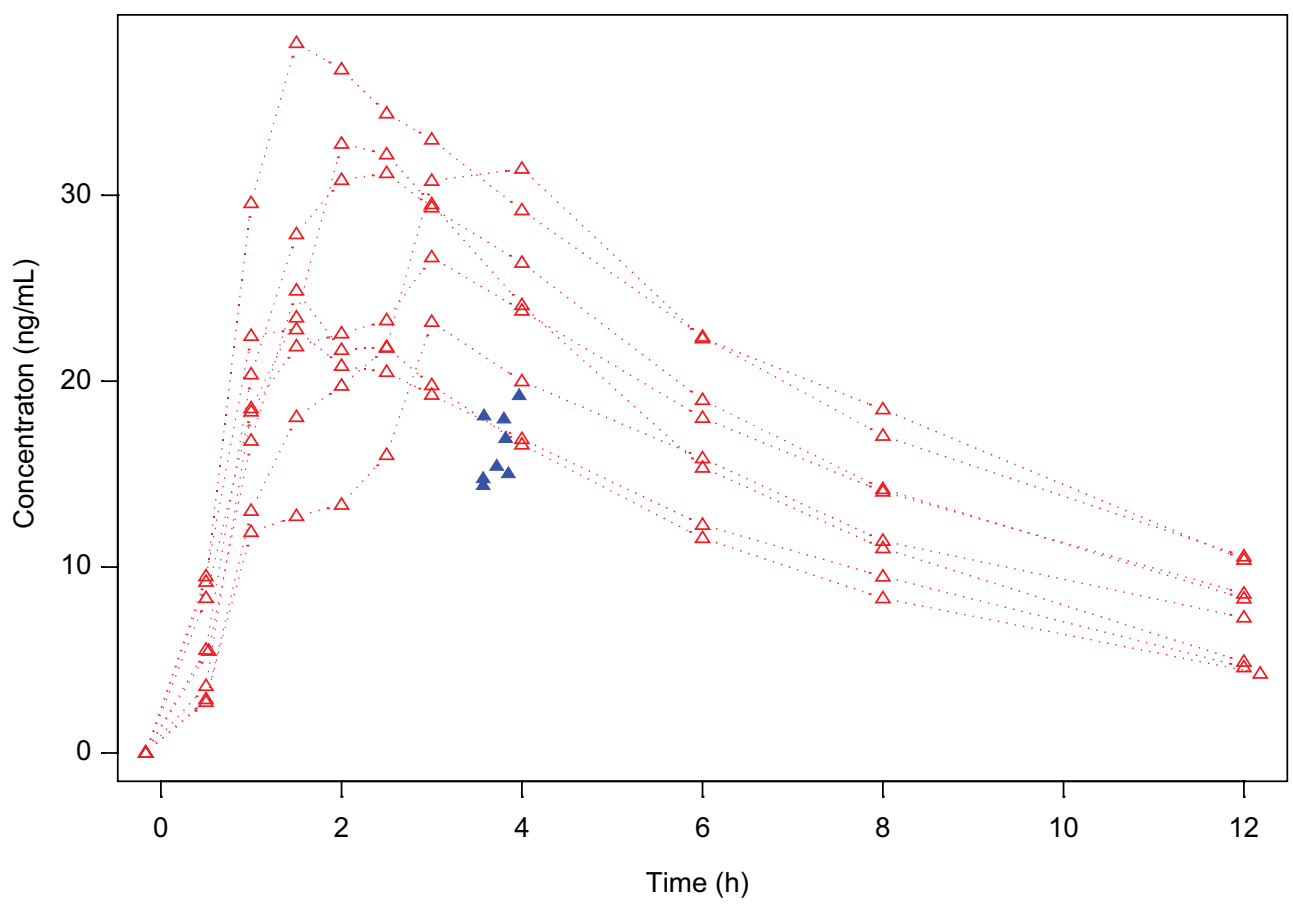

Figure 2 Individual pomalidomide plasma concentration (red lines) and semen concentration (blue triangles) profiles following multiple-dose administration.

Table 3 Individual pomalidomide semen PK (2 mg cohort)

\begin{tabular}{llllll}
\hline ID & $\begin{array}{l}\text { Actual semen } \\
\text { sampling time } \\
\text { (hours after dose) }\end{array}$ & $\begin{array}{l}\text { Semen } \\
\text { concentration } \\
\text { (ng/mL) }\end{array}$ & $\begin{array}{l}\text { Total net weight of } \\
\text { semen samples (g) }\end{array}$ & $\begin{array}{l}\text { Amount of pomalidomide } \\
\text { excreted in semen (ng) }\end{array}$ & $\begin{array}{l}\text { Percentage of } \\
\text { dose excreted in } \\
\text { ejaculation (\%) }\end{array}$ \\
\hline 301 & 3.97 & 19.2 & 2.2 & 42.3 & 0.0021 \\
303 & 3.72 & 15.4 & 3.6 & 56.0 & 0.0028 \\
305 & 3.57 & 14.8 & 0.7 & 10.5 & 0.0005 \\
306 & 3.82 & 17.0 & 1.4 & 24.4 & 0.0012 \\
307 & 3.57 & 14.4 & 2.1 & 30.1 & 0.0015 \\
308 & 3.58 & 18.1 & 1.6 & 28.7 & 0.0014 \\
310 & 3.8 & 18.0 & 6.3 & 113.1 & 0.0057 \\
311 & 3.85 & 15.0 & 2.6 & 39.7 & 0.0020 \\
\hline
\end{tabular}

Abbreviation: PK, pharmacokinetics.

physiology and morphology, data on the distribution of drugs into the seminal compartment are scarce and incomplete. Exact mechanisms of distribution are currently unknown, and conflicting data exist. ${ }^{22-24}$ Pomalidomide has showed good distribution properties. In the preclinical studies, it was shown that following a single oral administration of $\left[{ }^{14} \mathrm{C}\right]$-pomalidomide to pigmented male Long-Evans rats, compound-derived radioactivity was rapidly $\left(t_{\max }\right.$ of 3 hours in most tissues) distributed into various tissues and organs, but tissue and organ concentrations were below quantifiable levels by 24 hours after dosing (data on file). The highest measured tissue concentrations occurred in the gastrointestinal tract, bile, and kidney. Moderate concentrations were found in most tissues, including the liver, endocrine glands, secretory glands, and pigmented skin. Following administration to pregnant rabbits, pomalidomide-derived radioactivity was also observed in the spinal cord, brain, and blood of the rabbit fetuses, indicating it crosses the placenta in rabbits. ${ }^{25}$ Together, these data indicate good distribution of pomalidomide-related material into most tissues. Therefore, whether pomalidomide can distribute into semen in humans, the quantity of pomalidomide in each ejaculate, and the duration of the drug in semen are critical clinically relevant concerns to address considering the possible risk of embryo/ fetal exposure to pomalidomide that might be absorbed from ejaculate in females of childbearing potential.

In a previous semen distribution study with pomalidomide's analog lenalidomide, ${ }^{16}$ the lenalidomide concentration 

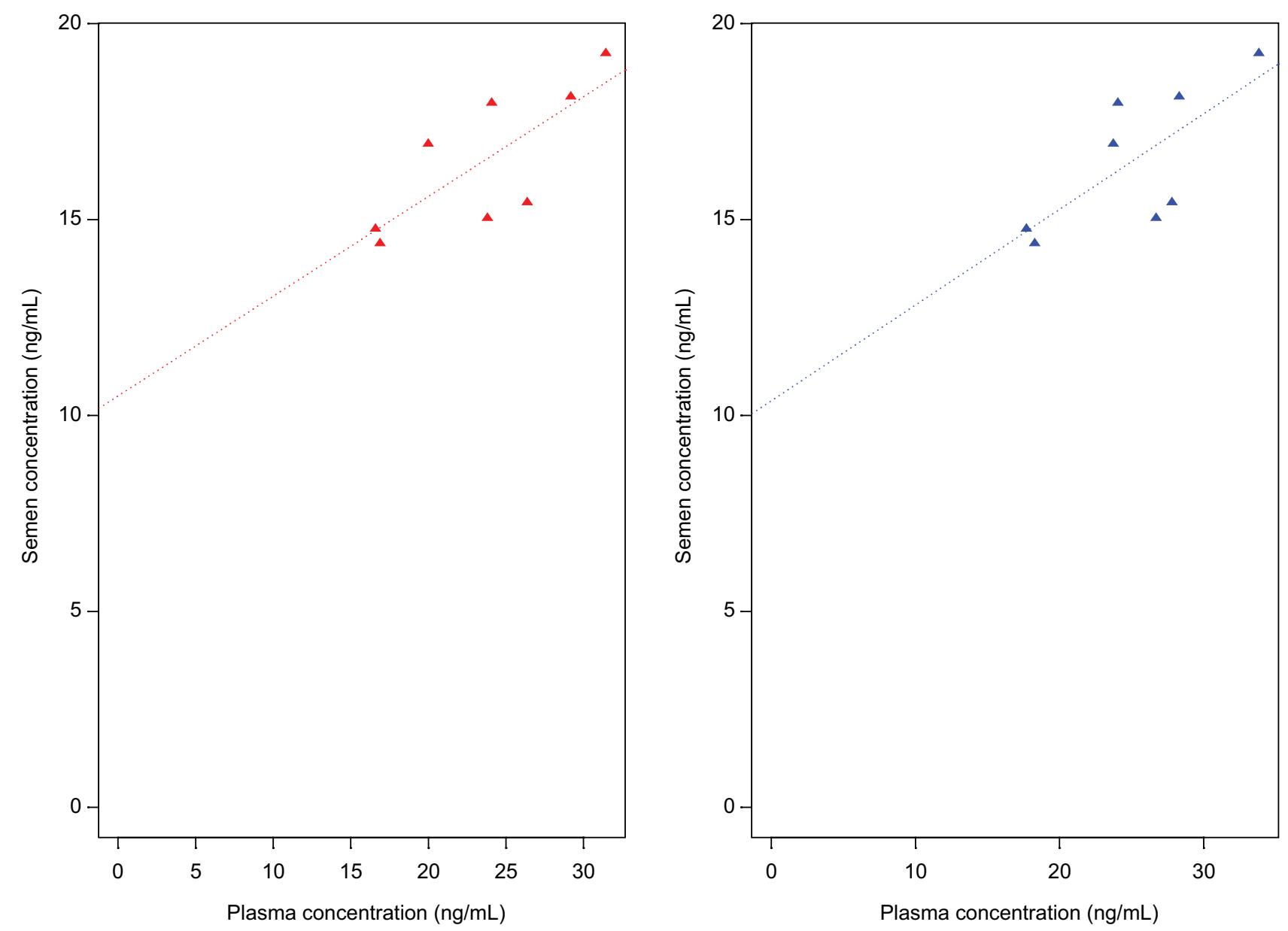

Figure 3 Correlation between plasma pomalidomide concentrations (4 hours after Day I dose [left panel] and 4 hours after Day 5 dose [right panel]) versus semen pomalidomide concentrations (4 hours after Day 4 dose).

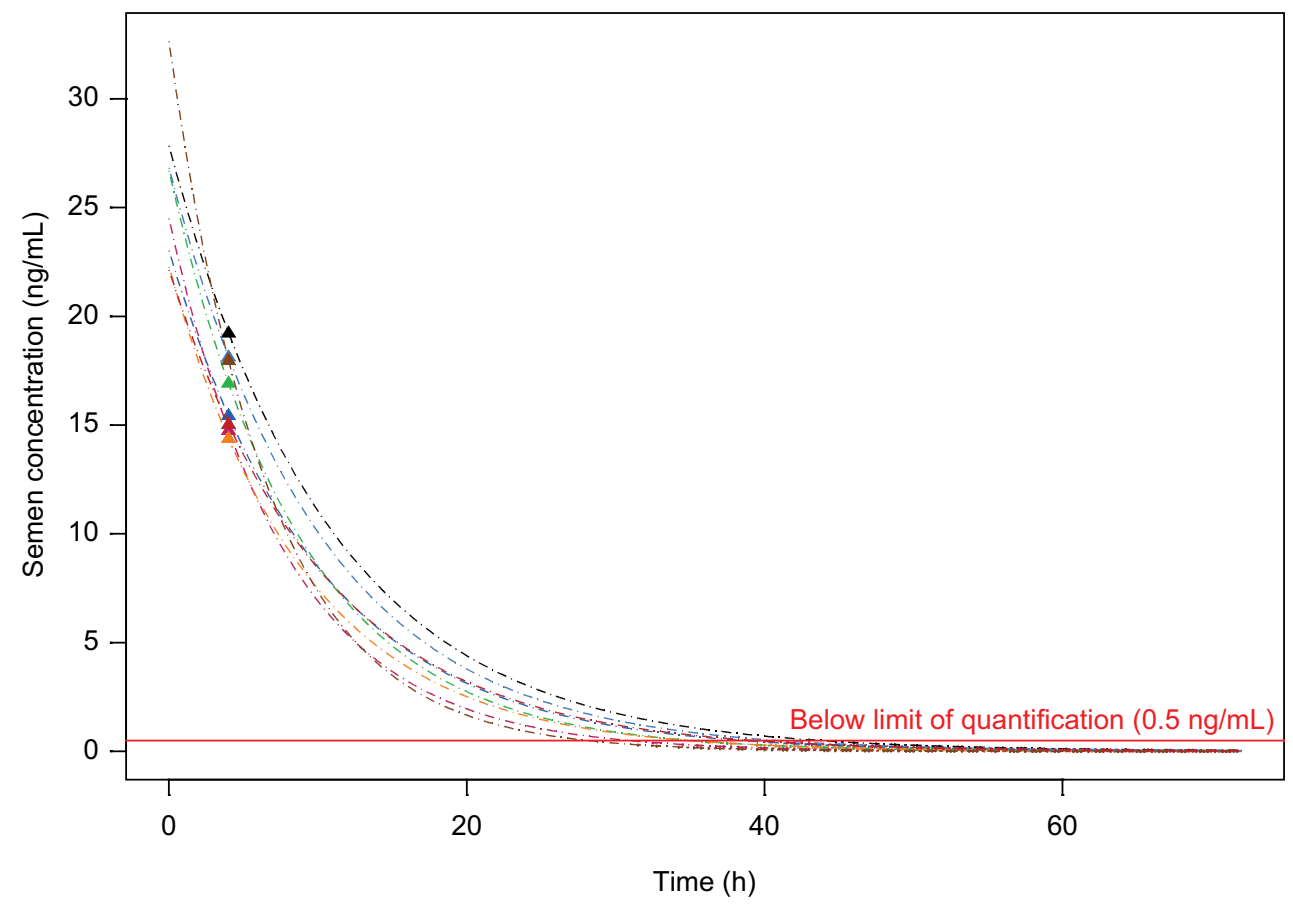

Figure 4 Simulation of semen pomalidomide concentration profiles assuming the elimination rate constant from the semen is the same as that from the plasma. 
in semen was $478 \mathrm{ng} / \mathrm{mL}$ at 2 hours and $10.0 \mathrm{ng} / \mathrm{mL}$ at 24 hours, which was higher than the corresponding drug concentration in plasma $(219 \mathrm{ng} / \mathrm{mL}$ at 2 hours and undetectable at 24 hours) but roughly paralleled the time course for plasma drug elimination. The mean amount of lenalidomide was $1,379 \mathrm{ng}$ per ejaculate at 2 hours and $35 \mathrm{ng}$ per ejaculate at 24 hours. The maximal drug content in a single ejaculate was $<2,000 \mathrm{ng}(<0.01 \%$ of the daily $25 \mathrm{mg}$ dose $)$. Lenalidomide was undetectable in semen at 72 and 168 hours. Considering the similarity in chemical structure and risk of embryo/fetal exposure to drugs through semen transmission, assessing the pomalidomide semen distribution was one of the main PK objectives of our study.

The pomalidomide concentration in semen 4 hours after dosing was approximately $67.0 \%$ of the plasma concentration at the same time point, the average amount of pomalidomide ejaculated at 4 hours was equivalent to $0.0022 \%$ of the daily dose, and the maximum amount of pomalidomide ejaculated at 4 hours from the 8 subjects was equivalent to $0.0057 \%$ of the daily dose, which accounts for only a very small portion of the daily dose (<120 ng). In general, there was a good correlation between the plasma concentrations and semen concentrations, that is, the subjects who had higher plasma pomalidomide concentrations tended to have higher semen pomalidomide concentration. Because semen samples from the 8 subjects who received the $2 \mathrm{mg}$ dose were collected at only one time point, it is challenging to assess how long pomalidomide was present in the semen. However, in the previous lenalidomide semen distribution study, ${ }^{16}$ in which semen samples were collected at multiple time points, it was shown that the elimination rate constants from lenalidomide semen samples were similar to those from plasma samples, and the overall elimination profile for lenalidomide in seminal reservoirs roughly paralleled that in plasma, suggesting that the plasma drug concentration may be the main driving force for the distribution of lenalidomide into seminal reservoirs. In addition, results from a rat quantitative whole-body autoradiography study showed that pomalidomide-derived radioactivity was rapidly and widely distributed into various reproductive tissues (epididymis, prostate gland, and seminal vesicles), but tissue concentrations were below the lower limit of quantitation 12 hours after dosing, suggesting there is no drug accumulation in reproductive tissues. Furthermore, the pomalidomide-derived radioactivity profiles in various reproductive tissues have a profile similar to that of the plasma concentration, especially at the terminal elimination phase, suggesting that like lenalidomide, the pomalidomide plasma concentration may be the main driving force for the distribution of pomalidomide into seminal reservoirs. As a result, the simulation of the semen concentration profiles conducted assumed the same elimination rate constant from semen samples as that from the plasma samples. The simulation indicated that none of the 8 study subjects who provided a semen sample would have had a detectable drug concentration in their semen beyond 48 hours after dosing (Figure 4). Finally, following multiple dosing, pomalidomide concentrations appeared to be at steady state by Day 3, and slight increases (up to $14.0 \%$ ) in $C_{\max }$ and $\mathrm{AUC}_{0-24}$ were observed Day 5 compared with Day 1. Similar low levels of accumulation were observed in patients with multiple myeloma who received $4 \mathrm{mg}$ pomalidomide QD for 1 week or $5 \mathrm{mg}$ QD for 4 weeks; ${ }^{11,12}$ therefore, a significant accumulation of pomalidomide in semen reservoirs after multiple doses is unlikely.

In assessing the risk of the pomalidomide transmission via semen to the embryo or fetus, the amount of pomalidomide exposure in nonphysical barrier-protected sexual intercourse between female and male partners is the key parameter, and challenging to estimate. Physiologically, pomalidomide has to distribute or permeate through several barriers to access the embryo and/or fetus. Passing through each barrier will decrease the pomalidomide exposure by dilution and redistribution. Based on the maximal pomalidomide amount that we measured in a single ejaculate 4 hours after dosing (approximately $0.0057 \%$ of the daily dose administered), the pomalidomide exposure in a female partner and subsequently in an embryo or fetus would be more than 4 orders of magnitude lower than the plasma exposure in the treated male subjects (assuming that $100 \%$ of the drug in semen is absorbed from the vagina).

\section{Conclusion}

Our study found pomalidomide to be present in male subjects' semen samples. The average amount of pomalidomide 4 hours after dosing in a single ejaculate is $<0.0022 \%$ of the daily $2 \mathrm{mg}$ dose, and simulation results suggest that pomalidomide would have been undetectable in semen 48 hours after stopping dosing. Based on the study results, the pomalidomide prescribing information approved by US Food and Drug Administration states that "pomalidomide is present in the semen of patients receiving the drug. Therefore, males must always use a latex or synthetic condom during any sexual contact with females of reproductive potential while taking POMALYST and for up to 4 weeks after discontinuing POMALYST, even if they have undergone a successful vasectomy. Male patients taking POMALYST must not donate sperm." 


\section{Disclosure}

Yan Li, Xiaomin Wang, Liangang Liu, Josephine Reyes, Maria Palmisano, and Simon Zhou are employees of and hold equity ownership in Celgene Corporation. The authors report no other conflicts of interest in this work.

\section{References}

1. Mitsiades N, Mitsiades CS, Poulaki V, et al. Apoptotic signaling induced by immunomodulatory thalidomide analogs in human multiple myeloma cells: therapeutic implications. Blood. 2002;99(12):4525-4530.

2. Zhu D, Corral LG, Fleming YW, Stein B. Immunomodulatory drugs Revlimid (lenalidomide) and CC-4047 induce apoptosis of both hematological and solid tumor cells through NK cell activation. Cancer Immunol Immunother. 2008;57(12):1849-1859.

3. Gupta D, Treon SP, Shima Y, et al. Adherence of multiple myeloma cells to bone marrow stromal cells upregulates vascular endothelial growth factor secretion: therapeutic applications. Leukemia. 2001;15(12):1950-1961.

4. Hideshima T, Chauhan D, Shima Y, et al. Thalidomide and its analogs overcome drug resistance of human multiple myeloma cells to conventional therapy. Blood. 2000;96(9):2943-2950.

5. Lu L, Payvandi F, Wu L, et al. The anti-cancer drug lenalidomide inhibits angiogenesis and metastasis via multiple inhibitory effects on endothelial cell function in normoxic and hypoxic conditions. Microvasc Res. 2009;77(2):78-86.

6. Reddy N, Hernandez-Ilizaliturri FJ, Deeb G, et al. Immunomodulatory drugs stimulate natural killer-cell function, alter cytokine production by dendritic cells, and inhibit angiogenesis enhancing the anti-tumour activity of rituximab in vivo. Br J Haematol. 2008;140(1):36-45.

7. Verhelle D, Corral LG, Wong K, et al. Lenalidomide and CC-4047 inhibit the proliferation of malignant $\mathrm{B}$ cells while expanding normal CD34+ progenitor cells. Cancer Res. 2007;67(2):746-755.

8. Corral LG, Haslett PA, Muller GW, et al. Differential cytokine modulation and $\mathrm{T}$ cell activation by two distinct classes of thalidomide analogues that are potent inhibitors of TNF- $\alpha$. J Immunol. 1999;163(1): 380-386.

9. Hayashi T, Hideshima T, Akiyama M, et al. Molecular mechanisms whereby immunomodulatory drugs activate natural killer cells: clinical application. Br J Haematol. 2005;128(2):192-203.
10. Teo SK, Chen Y, Muller GW, et al. Chiral inversion of the second generation IMiD CC-4047 (ACTIMID) in human plasma and phosphatebuffered saline. Chirality. 2003;15(4):348-351.

11. Imnovid (pomalidomide) [summary of product characteristics]. Celgene Europe; Uxbridge, UK, 2016.

12. POMALYST ${ }^{\circledR}$ (pomalidomide) PI [package insert]. Summit, NJ: Celgene Corporation; 2016.

13. Vargesson N. Thalidomide-induced teratogenesis: history and mechanisms. Birth Defects Res C Embryo Today. 2015;105(2):140-156.

14. Therapontos C, Erskine L, Gardner ER, Figg WD, Vargesson N. Thalidomide induces limb defects by preventing angiogenic outgrowth during early limb formation. Proc Natl Acad Sci U SA. 2009;106(21):8573-8578.

15. Ito T, Ando H, Handa H. Teratogenic effects of thalidomide: molecular mechanisms. Cell Mol Life Sci. 2011;68(9):1569-1579.

16. Chen N, Lau H, Choudhury S, Wang X, Assaf M, Laskin OL. Distribution of lenalidomide into semen of healthy men after multiple oral doses. J Clin Pharmacol. 2010;50(7):767-774.

17. Lutwak-Mann C, Schmid K, Keberle H. Thalidomide in rabbit semen. Nature. 1967;214(5092):1018-1020.

18. Hui JY, Hoffmann M, Kumar G. Embryo-fetal exposure and developmental outcome of thalidomide following oral and intravaginal administration to pregnant rabbits. Reprod Toxicol. 2014;48:115-123.

19. Teo SK, Harden JL, Burke AB, et al. Thalidomide is distributed into human semen after oral dosing. Drug Metab Dispos. 2001;29(10): $1355-1357$

20. Hoffmann M, Kasserra C, Reyes J, et al. Absorption, metabolism and excretion of $\left[{ }^{14} \mathrm{C}\right]$ pomalidomide in humans following oral administration. Cancer Chemother Pharmacol. 2013;71(2):489-501.

21. D'Amato RJ, Loughnan, Flynn E FJ. Thalidomide is an inhibitor of angiogenesis. Proc Natl Acad Sci U SA. 1994;91(9):4082-4085.

22. Kashuba AD, Dyer JR, Kramer LM, Raasch RH, Eron JJ, Cohen MS. Antiretroviral-drug concentrations in semen: implications for sexual transmission of human immunodeficiency virus type 1 . Antimicrob Agents Chemother. 1999;43(8):1817-1826.

23. Pichini S, Zuccaro P, Pacifici R. Drugs in semen. Clin Pharmacokinet. 1994;26(5):356-373.

24. Armstrong JR, Cook FE, Robison JR. Concentration of antibiotic and chemotherapeutic agents in the ejaculum. J Urol. 1968;100(1):72-76.

25. EMEA. European Medicines Agency Assessment Report; 2013. Available from: http://wwwemaeuropaeu/docs/en_GB/document_library/ EPAR__Public_assessment_report/human/002682/WC500147721pdf. Accessed June 22, 2017.
Clinical Pharmacology: Advances and Applications

\section{Publish your work in this journal}

Clinical Pharmacology: Advances and Applications is an international, peer-reviewed, open access journal publishing original research, reports, reviews and commentaries on all areas of drug experience in humans. The manuscript management system is completely online and includes a very quick and fair peer-review system, which is all easy to use.

\section{Dovepress}

Visit http://www.dovepress.com/testimonials.php to read real quotes from published authors. 